\title{
Influence of contact resistance on shielding efficiency of shielding gutters for $\mathrm{HV}$ cables
}

\author{
S. Koroglu ${ }^{1}$, P. Sergeant ${ }^{2,3}$, R. Sabariego ${ }^{4}$ Vuong Q. Dang ${ }^{4}$ and M. De Wulf ${ }^{5}$ \\ ${ }^{1}$ Dept. Electrical Engineering, Yildiz Technical University, 34349, Besiktas-Istanbul, Turkey \\ ${ }^{2}$ Dept. Electrical Energy, Systems and Automation, Ghent University, B-9000 Ghent, Belgium \\ ${ }^{3}$ Dept. Electrotechnology, Faculty Applied Engineering Sciences, University College Ghent, B-9000 Ghent \\ ${ }^{4}$ Dept. Electrical Engineering \& Computer Science, ACE, B-4000 LIEGE, Belgium \\ ${ }^{5}$ ArcelorMittal Global R\&D Gent, B-9060 Zelzate, Belgium \\ Peter.sergeant@ugent.be
}

\begin{abstract}
Shielding of buried three phase high voltage cables can be done by placing the cables in conducting ferromagnetic $U$ shaped gutters covered by plates. In case of a perfect electrical contact between adjacent gutters and between adjacent cover plates, the induced currents in the shield efficiently reduce the field generated in the vicinity of the cables. However, as a perfect contact cannot be guaranteed, it is useful to quantify the effect of a bad electrical contact on the shielding performance. From 2D FEM, 3D FEM and experiments, it is observed that a bad contact does not influence significantly the shielding if the axial length of the plates is relatively long compared to their other dimensions.
\end{abstract}

\section{ShiElding OF High VOlTAGE CABLES}

The U-shaped gutter elements (see Fig. 1) in which the three phase high voltage (HV) cables are buried, have a slightly conical shape in the axial direction. This shape allows stacking adjacent pieces with a small overlap to improve the electrical contact, which clearly favors an optimal distribution of the induced currents in the gutter and by consequence a good shielding. Sometimes the gutters are connected with clips to reduce the contact resistance, but they are usually not welded to each other. Also the cover plates are stacked with overlap, but this does not guarantee a good contact during the whole lifetime of the power line.

\section{NUMERICAL MODELS AND EXPERIMENTS}

To study if the shield works properly in case of bad electrical contact, we consider three approaches:

- 2D FEM in the $x y$ plane (Fig. 1) coupled with a small electric circuit that takes into account the extra resistance and a single valued nonlinear $B H$-curve.

- 3D FEM with thin shell formulation that models the overlapping zones and the contact resistances [1].

- Experimental setup consisting of three bus bars with adjustable balanced three phase currents up to $500 \mathrm{~A} \mathrm{rms}$, a $3 \mathrm{~mm}$ thick shield in hot rolled galvanized material (ArcelorMittal) and a magnetic field measuring system.

The results of the measurements and the simulations in Fig. 2 show that at $1 \mathrm{~m}$ height $(y=1 \mathrm{~m})$, the flux density is reduced by on average a factor of 2.5 with only the gutter and by a factor 6 when combining the gutter and cover plate $(\mathrm{CP})$.

\section{EFFECT OF THE CONTACT RESISTANCE}

According to Fig. 2, the difference between measured flux density with good contact (overlap and clips) and bad contact (plastic foil between adjacent gutters) is rather small, while the effect in case of only cover plates is large [2]. However, the gutters have a large ratio of axial length $(1.2 \mathrm{~m})$ to height
$(0.27 \mathrm{~m})$. For gutters with a small ratio, a bad contact deteriorates more the shielding performance. In the full paper, the $2 \mathrm{D}$ and $3 \mathrm{D}$ FE models will be accurately validated for several shielding configurations. Further, the influence of the amplitude of the currents in the cables will be quantified-it is shown in [2] that this has an effect because of the nonlinear magnetic characteristic - and of the dimensions of the gutters.

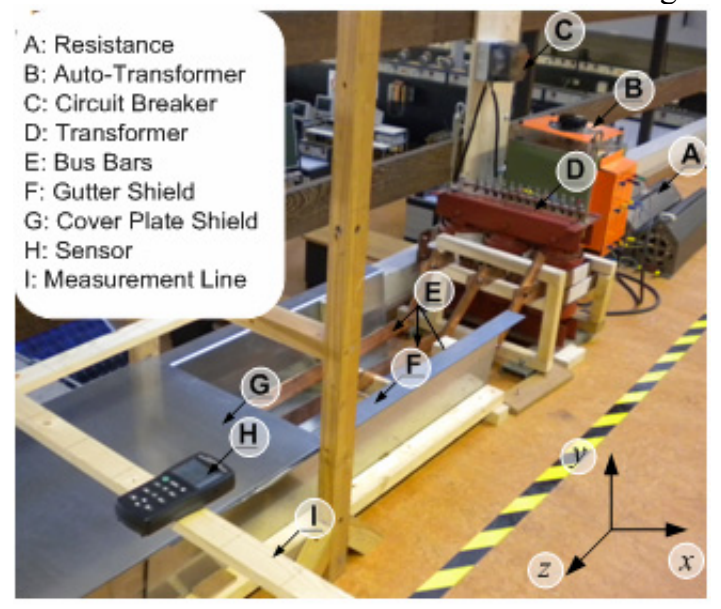

Fig. 1 Experimental set-up and $x y z$-reference frame. The origin of the $x y z$-reference frame is at the middle of the bottom of the gutter.

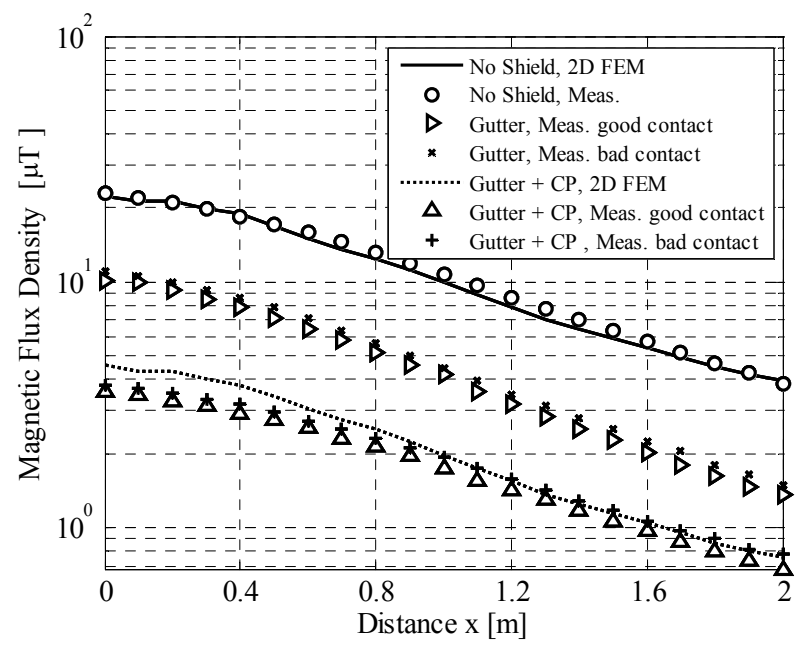

Fig. 2 Flux density at $y=1 \mathrm{~m}$ for several shield configurations and 500 A rms

\section{REFERENCES}

[1] C. Geuzaine, P. Dular, and W. Legros, "Dual formulations for the modeling of thin electromagnetic shells using edge elements," IEEE Trans. Magn., Vol. 36, No. 4, Jul. 2000.

[2] P. Sergeant, L. Dupré and J. Melkebeek, "Magnetic shielding of buried high voltage cables by conductive metal plates", COMPEL, Vol. 27, No. 1, pp. 170-180, 2008. 